\title{
Letter inception - a systematic review quantifying letters to the editor by medical students
}

\author{
Leeves, $L$. \\ King's College London, United Kingdom
}

\begin{abstract}
Background:
Letters to the Editor offer a way for readers to engage with authors on their publications and add valuable insights into the discussion. Letters are the shortest manuscript for medical students to publish and medical education journals are best suited for this. The UK Foundation Programme rewards medical students for achieving publications with a PubMed ID. The aim of the study is to analyse the total number, percentage, and affiliation of Letters to the Editor published by medical students in medical education journals.
\end{abstract}

\section{Methods:}

A review of 15 medical education journals that had an impact factor was conducted to identify the number and percentage of Letters to the Editor by medical students between July 2018 and June 2020. Additionally, information on the affiliation of the medical students was collected.

\section{Results:}

Over a two-year period, 299 letters were published of which $45.9 \%$ were by medical students. There was a $60 \%$ overall increase in letters by medical students published in the first 12 months compared to second 12 months. During this same period the overall number of letters published increased by $27 \%$. There was no apparent seasonal variation observed in Letters to the Editor published by medical students. A large proportion of letters by medical students were contributed by a small number of medical schools with five schools accounting for $60.5 \%$ of letters during the two-year period. The three medical schools with the highest number of letters published were King's College London, Imperial College London and University of Oxford for both 2018/19 and 2019/20. Most Letters to the Editor by medical students were by UK medical schools with $86 \%$ of letters published by UK medical students over the two-year period.

\section{Conclusion:}

There was an increase in the number of letters published overall but an even greater number published by students, which could represent greater awareness of letters to the editor as a means of dissemination amongst medical students. UK medical schools published many letters - it may be that this is due to the increasing importance in studentsl' minds about publications due to the impact on subsequent jobs when qualified. The results from our quantitative research shows a large number of letters are written by medical students, there is an increase in letters published from 2018/19 to 2019/20, and there is an overrepresentation of UK medical students - altogether this supports our hypothesis that medical students are publishing letters as a means of achieving a PubMed ID. Further qualitative research is required to prove our hypothesis. Journals should look for ways to encourage non-UK medical students to engage with their literature allowing for a more diverse range of opinions.

Keywords: Medical Education Publication 